\title{
COVID-19 Pandemic: Nursing Students' Clinical Education Challenges
}

\author{
Masoomeh Imanipour (iD) ${ }^{1}$ and Habib Shareinia (iD ${ }^{2, *}$ \\ ${ }^{1}$ Nursing and Midwifery Care Research Center, Critical Care and Management Department, School of Nursing and Midwifery, Tehran University of Medical Sciences, Tehran, \\ Iran \\ ${ }^{2}$ School of Nursing and Midwifery, Tehran University of Medical Sciences, Tehran, Iran \\ "Corresponding author: School of Nursing and Midwifery, Tehran University of Medical Sciences, Tehran, Iran. Email: habibshareinia@gmail.com
}

Received 2021 February 14; Revised 2021 May 25; Accepted 2021 May 26.

Keywords: Nursing Students, COVID-19 Pandemic, Nursing Education

\section{Dear editor,}

Clinical education is the most important part and the heart of professional nursing education. Nursing students can develop their theoretical knowledge by working in a clinical environment and gain sufficient proficiency (1). The acquisition of clinical skills and abilities requires students to gain experience in real environments with repetition and practice under the supervision of professors. It is estimated that clinical courses account for about 50\% of nursing bachelor's programs, which indicates their importance (2). The world health organization (WHO) has designated 2020 as the "International year of the nurse and the midwife", which is a unique opportunity to pay more attention to nursing education (3). Meanwhile, the outbreak of COVID-19 in late 2019 and its announcement as a pandemic in 2020 by the WHO has led to the closure of many universities around the world, including nursing schools, interrupting the theoretical and clinical training of students. Although theoretical training was resumed, though with many challenges, with the help of e-learning methods, how nursing students' clinical training courses are going to be conducted in hospitals is still problematic and ambiguous.

\section{Traditional Clinical Education Challenges}

Various studies show that the quality of clinical education to nursing students has not been good, facing challenges such as a large number of students, insufficient number of nursing professors experienced in the clinic, and the lack of space and educational facilities in hospitals (4-6). With the spread of COVID-19 and the issuance of preventive social distancing guidelines, nursing professors and planners faced yet another major challenge, namely the management of the number of the students attending clinical settings. In fact, a large number of students, the lack of clinical learning spaces, and the limited time to train each specialized course have made it difficult to reduce the number of students in each department to comply with health guidelines.

In addition, there is a concern that hospital administrators will not accept having nursing students in the clinic. This is especially true for freshmen who, due to being a beginner, cannot actively and effectively participate in real patient care and, in some ways, cause overcrowding and failure of social distancing.

The use of personal protective clothing by students and teachers, to be able to participate in clinical practice, and the lack of some facilities and related equipment in nursing schools are among other challenges and concerns of students and professors when attending hospitals. This is especially important for a number of special units and wards, such as intensive care units and infectious disease wards, where most COVID-19 patients are admitted.

On the other hand, during the COVID-19 outbreak, some wards have been shut down or dedicated to COVID19 patients. Also, a reduction in patients' referrals to clinics and hospitals due to fear of being infected, a reduction in elective treatments such as some surgeries, and even reduced number of accidents and traumas due to reduced travels and traffics between cities have led to fewer clinical cases for teaching students.

Students' and their families' worry about being in a hospital setting and the anxiety caused by the possibility of being infected or passing the disease on to the family are other issues that need attention and management. These issues affect the students living in dormitories in one way and the students living with their families in another.

\section{Virtual Clinical Education Challenges}

With the pandemic of COVID-19, and since the exact time of the disease's disappearance and its definitive treatment are unclear, it appears necessary for countries and so- 
cieties to try to live with the disease and resume their normal processes and lifestyles. Accordingly, it is necessary to consider measures for the educational processes of nursing students, especially in the field of clinical education. One way to do this is to move towards virtual clinical education. Virtual education and e-learning are among the topics raised in the education of medical students since many years ago and with advances in information technology and electronic tools, such as educational software, multimedia tools, web-based platforms, virtual education systems, virtual reality, and virtual patients. Although clinical education without directly facing the patient and the disease in real environments seems somewhat impossible, the use of virtual teaching methods as complementary techniques in clinical education can be effective in teaching practical skills and techniques to students. Despite the effectiveness of virtual clinical education, as confirmed in various studies (7-9), its implementation in the current situation is a challenge for some countries.

Although holding virtual rounds and clinical conferences will prevent the transfer of the clinical training load to hospital wards and conference rooms and allow for compliance with COVID-19 preventive guidelines, professors' unfamiliarity with how to conduct virtual clinical rounds and their inability to suddenly shift from conventional education to virtual education are the problems that most nursing schools face. Also, designing good and quality clinical scenarios that can transfer care concepts and nurture relevant skills in students through virtual education is a difficult task and requires teamwork.

Besides, the design of an interactive virtual clinic on the Internet, where students can practically face a clinical case, take all usual care procedures step by step, and receive feedback, requires the multidisciplinary collaboration of nursing and virtual education professors, as well as IT professionals. In addition, designing and preparing such a platform requires facilities and time.

Educational videos are another solution that can be used to promote clinical education amid the COVID-19 pandemic. In addition to shortening students' time of presence in the clinic, these videos can resolve the shortage of inadequate referrals of different clinical cases to hospitals and provide the opportunity to obtain the relevant knowledge in a safe environment without the possibility of being exposed to a disease or an infection. However, it should be noted that using some educational videos available on the Internet may not have the necessary educational effectiveness because they either do not fit the target audience or educational goals or lack the right educational design. Also, many standard educational videos are not available for free to all countries and all users or cannot be expected to be effective due to language barriers.
On the other hand, making educational videos that meet the educational needs and goals of the target audience and have the desirable educational design requires spending money, time, expertise, facilities, and multidisciplinary cooperation, which in the current situation may not be possible for all countries.

The novelty of the concepts of augmented reality and virtual reality in teaching clinical procedures and the lack of familiarity of all professors and educational managers with these concepts on the one hand, and the dependence of these technologies on factors such as special tools for design and use, their high costs, and being time consuming, on the other hand, have challenged the possibility of using these capabilities to improve students' professional qualifications in all countries and all nursing schools, which may be even impossible for some of them, at least in the current situation.

Issues such as countries' electronic infrastructure, Internet services, virtual learning infrastructure, lack of access of all students to the Internet, undesirable Internet speed, lack of access to required hardware such as laptops and smartphones by all students, and Internet costs can also be among the challenges related to the use of virtual clinical education.

Finally, nursing is a care-oriented discipline whose humanistic aspects are predominant and should be considered in education. Therefore, many professors and nursing professionals have questioned the ability of virtual clinical education in transferring many basic concepts of care and humanity, such as empathy, touch, and establishing effective communication, to nursing students.

\section{Conclusions}

The sudden emergence of the COVID-19 pandemic, which has led governments to impose restrictions to control the disease, has posed serious challenges to academic education. On the other hand, the rapid transmissibility of the disease, the lack of a definitive treatment for it, the need to follow preventive (e.g., using personal protective equipment) and social distancing guidelines, and the dependence of nursing students' education on hospital settings have made clinical education particularly difficult. The responsibility of educational authorities and nursing professors for the effective teaching of students while maintaining their health against the disease has left them with decision-making dichotomies. Overall, nursing schools need to address the issue of virtual clinical education; nevertheless, this itself is a new challenge for many countries. Therefore, reviewing curricula, reducing the duration of clinical education courses, changing clinical teaching and evaluation methods, establishing international communications to use other countries' experiences, providing free access to useful educational re- 
sources for all communities, governmental measures, and financial support for the development of virtual education, ensuring educational justice for all students, and plans to empower teachers in the field of virtual education can partly help face the challenges of nursing education amid the COVID-19 crisis. Despite all the challenges ahead, nursing educational authorities have to quickly develop short-term and long-term plans to shift from "post-COVID19 education" to "education amid COVID-19".

\section{Footnotes}

Authors' Contribution: M.I. and H.SH. participated in conceptualizing, designing, and drafting the manuscript.

Conflict of Interests: It was not declared by the authors. Funding/Support: It was not declared by the authors.

\section{References}

1. Papathanasiou IV, Tsaras K, Sarafis P. Views and perceptions of nursing students on their clinical learning environment: teaching and learning. Nurse Educ Today. 2014;34(1):57-60. doi: 10.1016/j.nedt.2013.02.007. [PubMed: 23481172].
2. Bjork IT, Berntsen K, Brynildsen G, Hestetun M. Nursing students' perceptions of their clinical learning environment in placements outside traditional hospital settings. J Clin Nurs. 2014;23(19-20):295867. doi: 10.1111/jocn.12532. [PubMed: 24460862]. [PubMed Central: PMC4263152].

3. Gennaro S. 2020: The Year of the Nurse as Seen Through a Coronavirus Lens.J Nurs Scholarsh. 2020;52(3):231-2. doi: 10.1111/jnu.12556. [PubMed: 32246789].

4. L'Ecuyer KM. Clinical education of nursing students with learning difficulties: An integrative review (part 1). Nurse Educ Pract. 2019;34:17384. doi: 10.1016/j.nepr.2018.11.015. [PubMed:30594118].

5. Jayasekara R, Smith C, Hall C, Rankin E, Smith M, Visvanathan V, et al. The effectiveness of clinical education models for undergraduate nursing programs: A systematic review. Nurse Educ Pract. 2018;29:11626. doi:10.1016/j.nepr.2017.12.006. [PubMed: 29272736].

6. Zhu Z, Xing W, Lizarondo L, Guo M, Hu Y. Nursing students' experiences with faculty incivility in the clinical education context: a qualitative systematic review and meta-synthesis. BMJ Open. 2019;9(2). e024383. doi: 10.1136/bmjopen-2018-024383. [PubMed: 30826795]. [PubMed Central: PMC6398780].

7. Hilty DM, Randhawa K, Maheu MM, McKean AJ, Pantera R, Mishkind MC. A review of telepresence, virtual reality, and augmented reality applied to clinical care.J Technol Behav Sci. 2020:1-28.

8. Forbes H, Oprescu FI, Downer T, Phillips NM, McTier L, Lord B, et al. Use of videos to support teaching and learning of clinical skills in nursing education: A review. Nurse Educ Today. 2016;42:53-6. doi: 10.1016/j.nedt.2016.04.010. [PubMed: 27237353].

9. Padilha JM, Machado PP, Ribeiro A, Ramos J, Costa P. Clinical Virtual Simulation in Nursing Education: Randomized Controlled Trial.JMed Internet Res. 2019;21(3). e11529. doi: 10.2196/11529. [PubMed: 30882355]. [PubMed Central: PMC6447149]. 